\title{
ONE DIMENSIONAL MODELLING OF FAILURE IN LAMINATED PLATES BY DELAMINATION BUCKLING
}

\author{
Herzl $\mathrm{Chal}^{\dagger}$, Charles D. Babcock $\ddagger$ and Wolfgang G. Knauss $\ddagger$ \\ Graduate Aeronautical Laboratories, California Institute of Technology, Mail Code 105-50, Pasadena, \\ CA 91125 , U.S.A
}

(Received 6 November 1980; in revised form 28 January 1981)

\begin{abstract}
When low speed objects impact composite laminated plates delamination may result. Under inplane compression such delaminations may buckle and tend to enlarge the delaminated area which can lead to loss of global plate stability.

This process is modelled here in a first attempt by a delaminating beam-column wherein the local delamination growth, stability and arrest are governed by a fracture mechanics-based energy release rate criterion.
\end{abstract}

\section{NOTATION}

$A_{3}$ midspane transverse deflection in Section 3

$D_{i} \frac{E t_{i}^{3}}{12\left(1-v^{2}\right)}$-bending rigidity in "ith" section

$E$ Young's modulus

$G$ strain energy release rate (s.e.r.r.) per unit area

$\tilde{G} \quad G\left\{\left\{E t^{5} L^{-4}\left(1-\nu^{2}\right)^{-1}\right\}\right.$

$G_{a} \ldots G_{e}$-s.e.r.r. associated with models $a \ldots e$

$h$ delamination thickness

$\bar{h} h / t$

$L$ total length of plate

1 delamination length

$\bar{i} / / L$

$l_{1}(L-I) / 2, l_{2}=l_{3}=1$

$I^{*} \| /\left\{h \Gamma_{0}^{*-1 / 4}\right\}$

$l_{0}$ initial film length

$l_{A}, l_{B}$ significant film length

$I_{0}^{*} l_{0}\left\{h \Gamma_{0}^{*-1 / 4}\right\}, I_{A}^{*}=3.376, I_{B}^{*}=2.221$

$I_{a^{*}}, I_{e^{*}}$ delamination length which maximize $G_{a}, G_{e}$ respect

$I_{a^{*}} I_{a^{*}} / L, I_{e^{*}}=I_{e^{*}} / L$

$P_{i}$ total load in "ith" section

$t$ total plate thickness

$t_{1} t, t_{2}=t-h, t_{3}=h$

$U$ strain energy

$U_{i}, U_{i,}, U_{u i}$ strain energy for three different states

$\bar{U} \quad U /\left\{E t^{9} L^{-3}\left(1-\nu^{2}\right)^{-1}\right\}$

$u_{i} \frac{l_{i}}{2} \vee\left(P_{i}\left(D_{i}\right), i=1,2,3\right.$ normalized total load in " $i t h "$ section

$\Gamma_{0}$ energy required to produce a unit of new surface

$\Gamma_{0}^{*} \Gamma_{0} /\left\{\frac{E h}{2\left(1-\nu^{2}\right)}\right\}$

$\delta$ end deflection of delamination

$\epsilon_{0}$ loading strain

$\epsilon_{i} \frac{P_{i}}{E t_{i}}, i=1,2,3$ midplane (membrane) strain in " $i$ th" section

$\epsilon_{L} \frac{\pi^{2}}{3\left(1-\nu^{2}\right)}\left(\frac{t}{L}\right)^{2}$ buckling strain of plate

$\epsilon_{\text {cr }} \frac{\pi^{2}}{3\left(1-\nu^{2}\right)}\left(\frac{h}{l}\right)^{2}$ buckling strain of delamination

$\bar{\epsilon}_{0} \epsilon_{0} / \epsilon_{L}, \bar{\epsilon}_{\mathrm{i}}=\epsilon_{\mathrm{J}} / \epsilon_{\mathrm{L}}, i=1,2,3, \bar{\epsilon}_{\mathrm{cr}}=\epsilon_{\mathrm{cr}} / \epsilon_{\mathrm{L}}=(\bar{h} / \bar{l})^{2}$

$\epsilon_{0}^{*}\left(1-\nu^{2}\right) \epsilon_{0} \Gamma_{0}^{*-1}$

$\epsilon_{0 A}^{*} \quad 0.866, \epsilon_{0}^{*}{ }_{B}=1.000$

$\eta \quad u_{3}-\pi$ expansion parameter

$\theta$ end rotation of delamination

$\bar{\theta} \quad \theta L / t$

к $1-\vec{h}+\vec{h} \vec{l}$

$\nu$ Poisson's ratio

†Graduate student and research assistant.

$\ddagger$ Professors of aeronautics. 


\section{INTRODUCTION}

Fiber reinforced plastics and, in particular, graphite fiber composite materials enjoy a definite strength to weight advantage over many standard engineering materials used in weight critical applications. This assessment must, however, be made with respect to applications where the primary stresses are aligned with the fiber direction such as the extension (tension or compression) or bending of a thin plate where stresses normal to the plane of the plate are small. If such a plate is subjected to impact, considerable damage can be caused since the cohesive strength of the plate through its thickness is quite low. This in turn can lead to degradation of the extensional or bending strength of the plate [1-5].

The mechanism of this strength degradation has been the subject of a recent investigation [6]. Although the details of the initial degradation process are poorly understood, it is believed that the strength degradation under compressive in-plane loading is the result of coupled delamination and delamination buckling.

An experimental investigation into the failure mechanism using high-speed photography[7] has shown that the failure process can be divided roughly into two phases. In the first phase the plate is impacted and the resulting response causes interlaminar separation. The size of this damage area is a function of the impactor parameters and the plate material, lay-up, etc,[6]. For the present discussion it will be assumed that the dimension of the damage area is large compared to the laminate thickness but small compared with the plate size.

In the second phase the damage area spreads to the undamaged area of the plate through a combination of laminate buckling and further delamination. It is this failure phase with which we are concerned in the following development. In order to elucidate the dominant physical phenomena in a readily tractable analytical manner it appears prudent to deal first with a geometrically simpler situation than the full plate problem illustrated in Fig. l: the treatment of that problem depends heavily on numerical computations. Instead we shall deal here only with the one dimensional plate analogue represented by the cross section in Fig. 1 which geometry and loading are considered to be invariant along the coordinate normal to the plane of the figure. In the subsequent analysis which is condensed from references [8-11] we shall start from the assumption that a delamination exists in the plate. The latter may be initially unloaded or under an in-plane compressive load when the delamination appears. In either case the analysis will study the growth (under load) of the damage area. Quasistatic conditions will be assumed and the analysis will draw on the theory of ordinary beams as well as a rate independent fracture criterion based on the energy release rate.

Growth of the delamination is assumed to occur in its own plane in keeping with the laminate character of layered composites. Yet, for simplicity reasons the properties of the plate are assumed homogeneous, isotropic, and linearly elastic. We note, however, that impact damage in a fiber composite of, say, quasi-isotropic and symmetric lay-up generates in general two or more delaminations none of which possess the same properties themselves. Such material behavior can be readily dealt with at the expense of introducing additional parameters into the problem; but, because neither the physical principles involved in the analysis nor the character of the results will change, we omit attention to that detail.

Depending on the thickness and number of delaminations relative to the total plate thickness several further approximations may be considered as illustrated in Fig. 2. In Fig. 2(a), the unbuckled portion of the plate has been made infinitely thick; this is called the "thin film" model. A finite thickness (assumed large compared to the delamination) is introduced in the delamination "thick column" model Fig. 2(b). The case of several delaminations can be analyzed (Fig. 2c) as well as a symmetrical split (Fig. 2d). The most general case analyzed in this report is represented in Fig. 2(e).

The analyses for all these models are delineated in this report. The "thin film" model is analyzed first since the results are quite simple and illustrative of the results for the more complete models $\dagger$.

†We wish to point out that after the typing of the manuscript we became aware of publications dealing with the thin film problem [12] as well as delamination of a ring under external pressure [13]. 


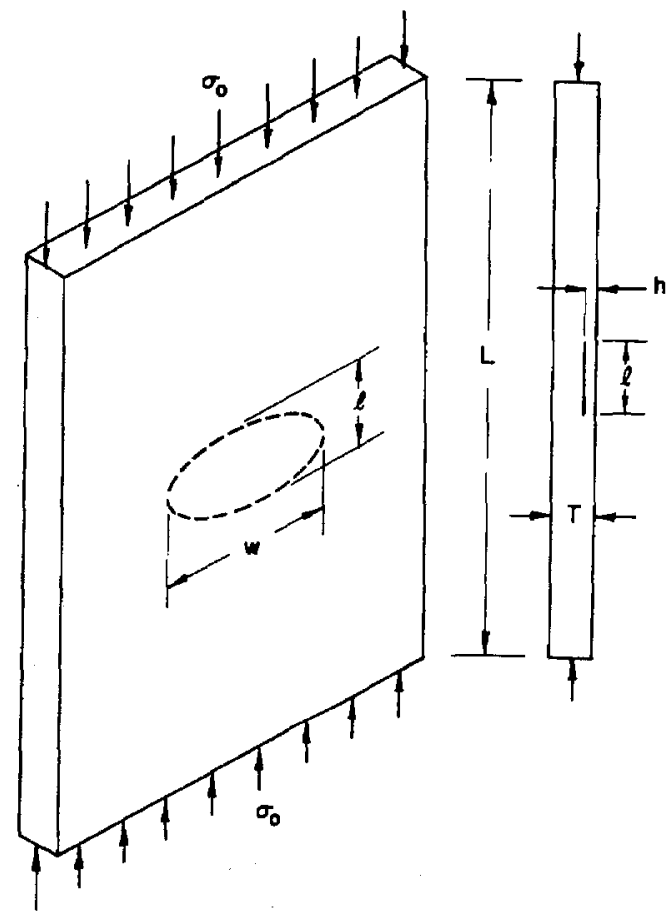

Fig. 1. Idealized delaminated plate.

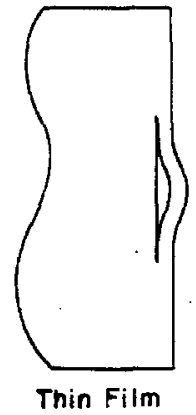

$\mathbf{a}$

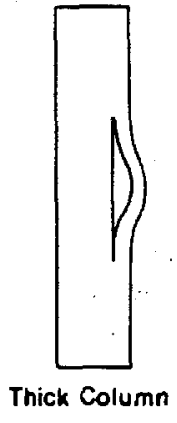

b

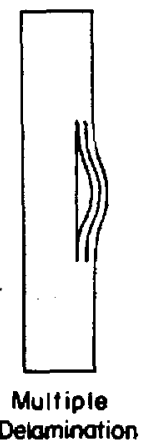

c
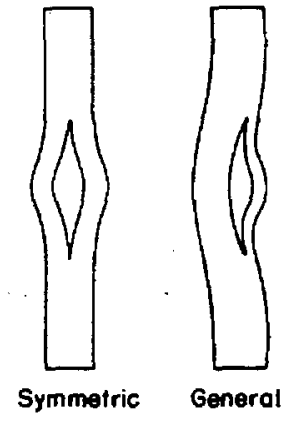

d

Fig. 2. Delamination/buckling models.

\section{EXAMPLE PROBLEM-"THIN FILM" DELAMINATION}

The stages in the "thin film" delamination and buckling are shown in Fig. 3. The delaminated film of thickness $h$ and length $l$ is part of an infinitely thick medium, characterized by Young's modulus, $E$, and Poisson's ratio $\nu$.

Under a compressive strain $\epsilon_{0}$, the delamination can grow only after the film buckles. In order for growth to occur, work of rupture is required. This energy must be available either from work done by the compression forces during the growth of the delamination (fixed load) and/or from the decrease of strain energy of the system. For the "thin film" delamination the strain in the backing medium, $\epsilon_{0}$, remains constant and all the energy available for crack growth is drawn from changes in stored strain energy caused by changes in the delamination length $l$. For this case (fixed grip), it is more convenient to write the "load" in terms of strain, $\epsilon_{0}$, rather than in terms of stress.

Consider the three stages i, ii and iii in Fig. 3. State i represents the unstressed medium while ii denotes the uniformly compressed medium (strain $=\epsilon_{0}$ ). State iii differs from ii in that 


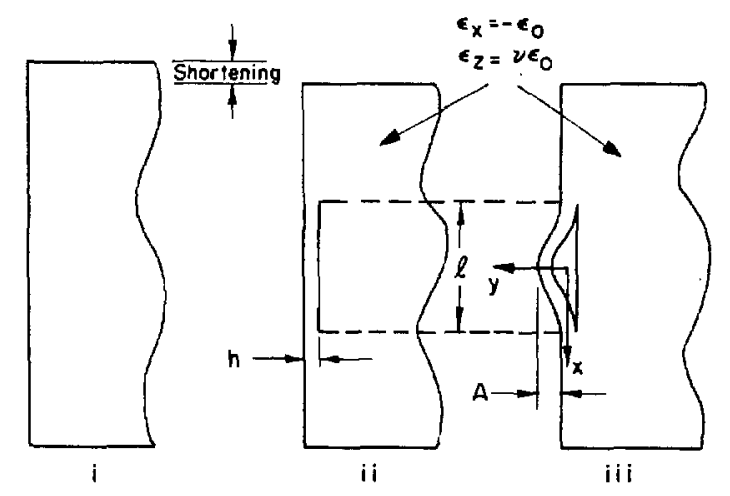

Fig. 3. Thin film model-three configurations.

the delamination has buckled. The conditions for growth of the delamination are determined by examining the change in stored energy of the system as the delamination spreads. If the elastic energy loss in that process equals or exceeds the energy required to create a unit of new delamination, then growth will take place. If growth does take place, it is of interest to examine whether or not the growth is arrested at a later stage.

The strain necessary to cause buckling, $\epsilon_{\mathrm{c}}$, can be easily calculated if use is made of the usual assumptions of technical beam/plate theory to yield,

$$
\epsilon_{\mathrm{cr}}=\frac{\pi^{2}}{3\left(1-\nu^{2}\right)}\left(\frac{h}{l}\right)^{2}
$$

The post buckled shape of the film can also be calculated assuming that the buckling displacement remains reasonably small.

$$
y=A \frac{1}{2}(1+\cos 2 \pi x / l)
$$

The amplitude $A$ is determined by the condition that in going from state ii to iii the length $l$ of the delaminated section remains unchanged, and the membrane stress in the buckled laminate is the same as the buckling stress. These conditions lead to

$$
\left(\epsilon_{0}-\epsilon_{\mathrm{cr}}\right)\left(1-\nu^{2}\right) l=\int_{-1 / 2}^{l / 2} \frac{1}{2}\left(\frac{\mathrm{d} y}{\mathrm{~d} x}\right)^{2} \mathrm{~d} x
$$

or

$$
A^{2}=\left(\epsilon_{0}-\epsilon_{\mathrm{cr}}\right)\left(\frac{2 l}{\pi}\right)^{2}\left(1-\nu^{2}\right)
$$

The strain energy in the buckled layer consists of the membrane energy and the bending energy. It is given by (on a per unit width basis).

$$
U_{\mathrm{iii}}=\frac{E h l}{2}\left[\epsilon_{\mathrm{cr}}^{2}\left(1-\nu^{2}\right)+\nu^{2} \epsilon_{0}^{2}\right]+\int_{-1 / 2}^{1 / 2} \frac{E h^{3}}{24\left(1-\nu^{2}\right)}\left(\frac{\mathrm{d}^{2} y}{\mathrm{~d} x^{2}}\right)^{2} \mathrm{~d} x
$$

or

$$
U_{\mathrm{iii}}=\frac{E h l\left(1-\nu^{2}\right)}{2}\left[2 \epsilon_{0} \epsilon_{\mathrm{cr}}-\epsilon_{\mathrm{cr}}^{2}+\frac{\nu^{2}}{1-\nu^{2}} \epsilon_{0}^{2}\right]
$$

Next the energy release rate, $G$, is calculated for the condition that the length of the film changes from $l$ to $l+\Delta l$. To distinguish the energy release rate in this example from those 
encountered later on let us affix a subscript " $a$ "; then

$$
G_{a}=\lim _{\Delta l \rightarrow 0}\left\{U_{\mathrm{iij}}(l)+\frac{1}{2} E \epsilon_{0}{ }^{2} h \Delta l-U_{\mathrm{iij}}(l+\Delta l)\right\} \frac{1}{\Delta l},
$$

which can be reduced to

$$
G_{a}=\frac{E h\left(1-\nu^{2}\right)}{2}\left(\epsilon_{0}-\epsilon_{\mathrm{cr}}\right)\left(\epsilon_{0}+3 \epsilon_{\mathrm{cr}}\right)
$$

Finally, the strain energy in the laminate corresponding to stage ii is calculated as

$$
U_{\mathrm{if}}=\frac{1}{2} E h \epsilon_{\epsilon_{0}}^{2}
$$

The history of the strain energy of the thin film delamination can now be considered. There will be two different cases depending upon the time in the load history at which the delamination is introduced. The first case considered will be the one in which the delamination is introduced prior to inception of loading. Next the case of a delamination introduced while the plate is under compression load will be considered.

For the first case the strain energy increases quadratically with load (eqn 9) until $\epsilon_{0}$ reaches $\epsilon_{\mathrm{cr}}$ (Fig. 4). At $\epsilon_{0}=\epsilon_{\mathrm{cr}}$ the laminate buckles and the strain energy increases in accordance with eqn (6). This is shown in Fig. 4 where $\nu$ has been chosen as 0.3 for plotting purposes.

Since the stresses at the ends of the delamination (the delamination crack-tip) are considered to promote further splitting only after buckling has occurred, the question of delamination stability is of interest only for $\epsilon_{0}>\epsilon_{\mathrm{cr}}$. Thus (8) indicates a positive strain energy release only for $\epsilon_{0}>\epsilon_{\mathrm{cr}}$. Whether further delamination occurs depends, however, on the magnitude of the fracture energy, $\Gamma_{0}$, which is defined as the energy required to produce a unit of new delamination. $t$ The dependence of the strain energy release rate, $G_{a}$, upon loading and delamination length (from (8)) is plotted in Fig. 5. In order to generalize these results for arbitrary Poisson's ratio $\nu$ and fracture energy $\Gamma_{0}$, the following normalizations were employed

$$
\begin{aligned}
\Gamma_{0}^{*} & =\Gamma_{0} /\left\{\frac{E h}{2\left(1-v^{2}\right)}\right\} \\
\epsilon_{0}^{*} & =\epsilon_{0} /\left\{\left(1-\nu^{2}\right)^{-1} \Gamma_{0}^{* 1 / 2}\right\} \\
l^{*} & =l /\left\{h \Gamma_{0}^{*-1 / 4}\right\} .
\end{aligned}
$$

With the aid of Fig. 5, the history of the delamination as a function of load can be determined. In this connection there are two values of load $\epsilon_{0}$ (or $\epsilon_{0}^{*}$ ) which have special significance: the first, denoted by $\epsilon_{0 A}$, corresponds to the lowest value of strain, for which the strain energy release rate can equal or exceed $\Gamma_{0}$. Referring to Fig. 5 it is clear that this value is determined from the dual condition

$$
G_{a}(l)=\Gamma_{0} \text { and } \frac{\partial G_{a}}{\partial l}=0
$$

which yields

$$
\epsilon_{0 A}=\frac{1}{2\left(1-\nu^{2}\right)} \sqrt{ }\left(3 \Gamma_{0}^{*}\right) \quad \text { or } \quad \epsilon_{0 A}^{*}=0.866
$$

and the corresponding delamination length

$$
l_{A}=h\left(2 \pi^{2} / \sqrt{ }(3)\right)^{1 / 2} \Gamma_{0}^{*-1 / 4} \text { or } l_{A}^{*}=3.376 .
$$

Note that a unit delamination generates two units of new fracture surface. If $\gamma_{0}$ is the fracture energy per unit of new surface then $\Gamma_{0}=2 \gamma_{0}$. The magnitude of $\Gamma_{0}$ has been measured for T300/5208 graphite epoxy to be $260 \mathrm{~N} / \mathrm{m}(1.5 \mathrm{lb} / \mathrm{in}) \pm$ $20 \%[8]$. 


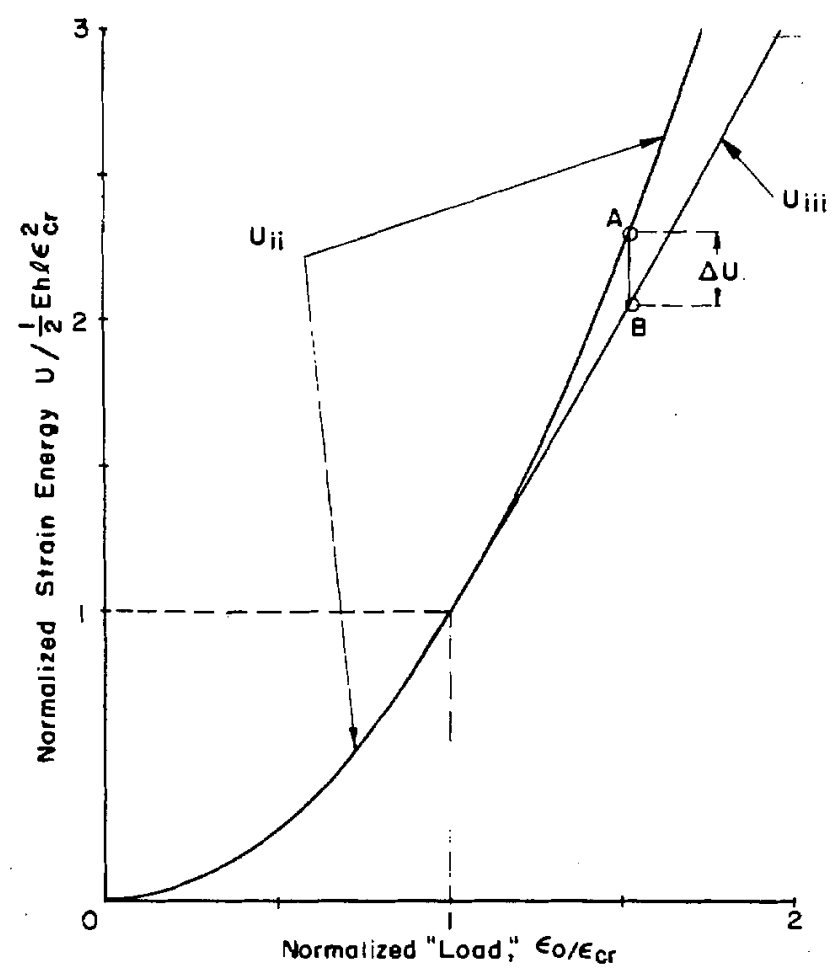

Fig. 4. Strain energy of the delaminated section as a function of load.

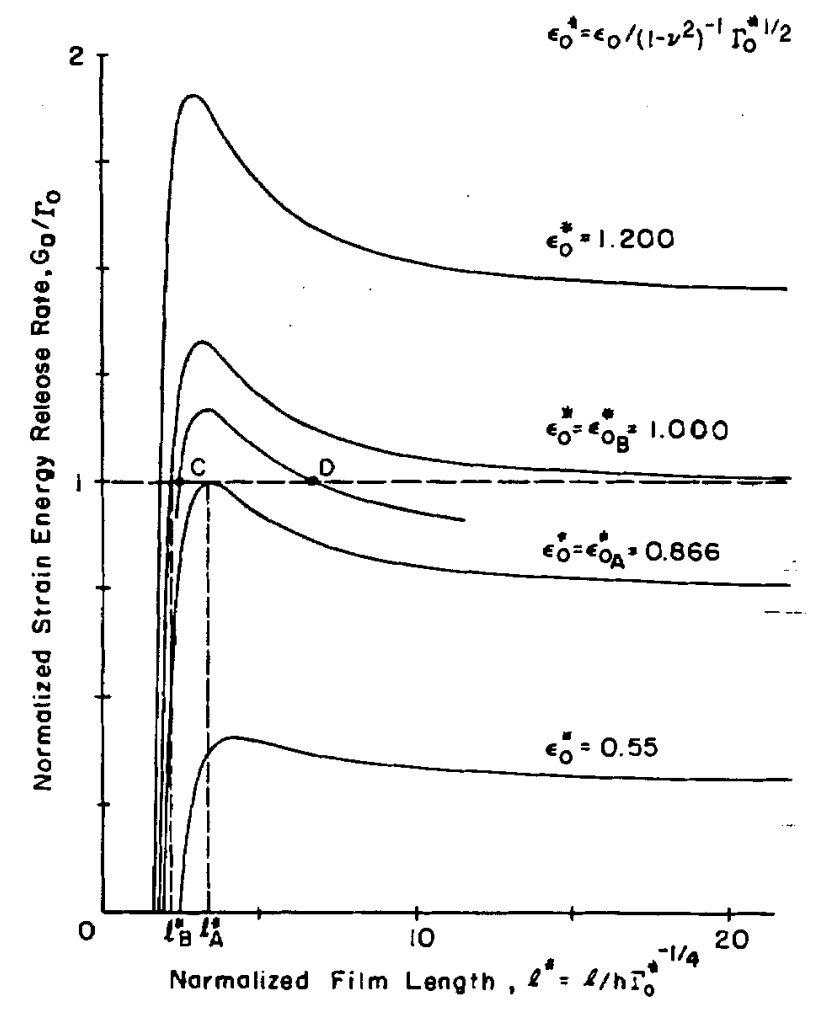

Fig. 5. Thin film model-strain energy release rate. 
The other important strain, denoted by $\epsilon_{0 B}$, is the limit beyond which $G_{a}$ exceeds $\Gamma_{0}$ as $l \rightarrow \infty$; it is given by

$$
\epsilon_{0 B}=\sqrt{ }\left(\Gamma_{0}^{*}\right) /\left(1-\nu^{2}\right) \text { or } \epsilon_{0 B}^{*}=1.000 .
$$

For this value of the load the strain energy release rate exceeds $\Gamma_{0}$ when the delamination length $l^{*}$ falls in the range

$$
l_{B}^{*}<l^{*}<\infty \quad\left(l_{B}=2.221\right) .
$$

By knowing these bounds, the length of the delaminated region can be found as a function of its initial length $l_{0}$ and the loading $\epsilon_{0}$. This is illustrated by Fig. 6 for normalized delaminated length and loading. Suppose the initial delamination length, $l_{0}$, is such that $l_{0}^{*}=l_{A}^{*}$, then a load $\epsilon_{0}^{*}$ increasing from zero will produce no further damage growth until $\epsilon_{0}^{*}=\epsilon_{0 A}^{*}$ in accordance with Fig. 6 (see path 1). When $\epsilon_{0}^{*}$ exceeds $\epsilon_{0 A}^{*}$ stable delamination growth occurs such that for $\epsilon_{0}^{*} \rightarrow \epsilon_{0 B}^{*}, l^{*} \rightarrow \infty$. It is also clear that for $l_{0}^{*}>l_{A}^{*}$ similarly stable growth occurs, though starting at values of $\epsilon_{o}^{*}$ larger than $\epsilon_{0 A}^{*}$ (see path 2). Now consider $l_{B}^{*}<l_{0}^{*}<l_{A}^{*}$ (see path 3 ). Then no growth occurs until $\epsilon_{0}^{*}$ is sufficiently large, corresponding to points $C$ in Figs. 5 and 6 . Thereafter unstable crack growth occurs until the delamination reaches a new length corresponding to point $D$ in Figs. 5 and 6, with only stable growth possible for a further increase in $\epsilon_{0}^{*}$. Finally, consider $l_{0}^{*}<l_{B}^{*}$. Then in accordance with path 4 in Fig. 6 no growth occurs until $\epsilon_{0}^{*}$ reaches a value larger than $\epsilon_{0}^{*}$, from which the delamination increases unstably to infinite length.

From this type of calculation it is clear that a variety of behavior may be observed during a test on a delaminated structure. The behavior would be dependent upon the dimension of the damaged area as well as on the other parameters of the problem.

For the second case let us consider the history of the strain energy if a delamination is introduced while the structure is under a load such that $\epsilon_{0}>\epsilon_{\mathrm{cr}}$. Prior to the introduction of the delamination the strain energy is given by (9). In Fig. 4, we associate with that strain the point A, say. Now introduce a delamination, and assume for the present that this process does not absorb energy from that stored in the system. Next buckling will occur and the new equilibrium state has a lower energy corresponding to point B on the branch of the energy trace marked $U_{\mathrm{iii}}$ in Fig. 4. If the energy state at that instant is such that $G_{A}>\Gamma_{0}$ further delamination will occur.

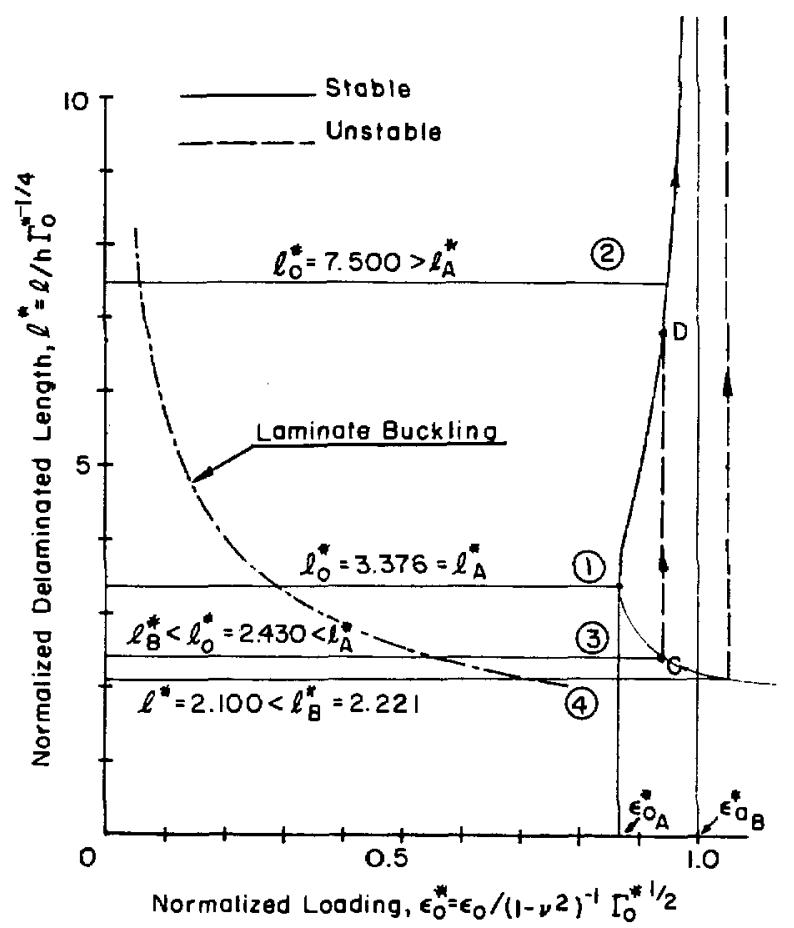

Fig. 6. Delaminated length as a function of load. 
However, note that even if the energy release rate at point $B$ in Fig. 4 is not sufficient to allow further delamination growth it may be possible that part of the energy released in the buckling process, denoted by $\Delta U$ in Fig. 4, contributes to the further fracture process. This possibility could be reflected in a lowering of the load at which the damage spreads if the delamination is introduced while the structure is under load, as compared to the case where delamination already existed prior to loading.

\section{GENERAL CASE}

Having considered the special and simple problem of film delamination let us now turn to the analysis for the "general" case (Fig. 2) which is developed along identical lines of reasoning as the thin film case. The algebra is more cumbersome and it is necessary to evaluate the energy release rate numerically. Cylindrical bending of the plate will be assumed along with a plane strain condition for the membrane stresses. The coordinate systems for the separate parts of the structure are shown in Fig. 7. Each section is treated as a beam column with compatibility and equilibrium enforced at the interfaces between sections. The specific conditions are:

\section{Compatibility}

Section 1

$$
\begin{aligned}
& y_{1}=\frac{\mathrm{d} y_{1}}{\mathrm{~d} x_{1}}=0 \quad \text { at } \quad x_{1}=0 \\
& y_{1}=\delta, \frac{\mathrm{d} y_{1}}{\mathrm{~d} x_{1}}=\theta \quad \text { at } \quad x_{1}=l_{1} .
\end{aligned}
$$

Section 2 and 3 (assume symmetry at $x_{i}=0$ )

$$
y_{i}=\delta, \frac{\mathrm{d} y_{i}}{\mathrm{~d} x_{i}}=\theta \quad \text { at } \quad x_{i}=-l_{i} / 2, i=2,3
$$

Use of these conditions and the solution to the beam column equation produces the following results:

$$
y_{i}(x)=f_{i}(x) \theta+g_{i}(x) \delta, i=1,2,3
$$

where $f_{i}$ and $g_{i}$ are given in the Appendix.

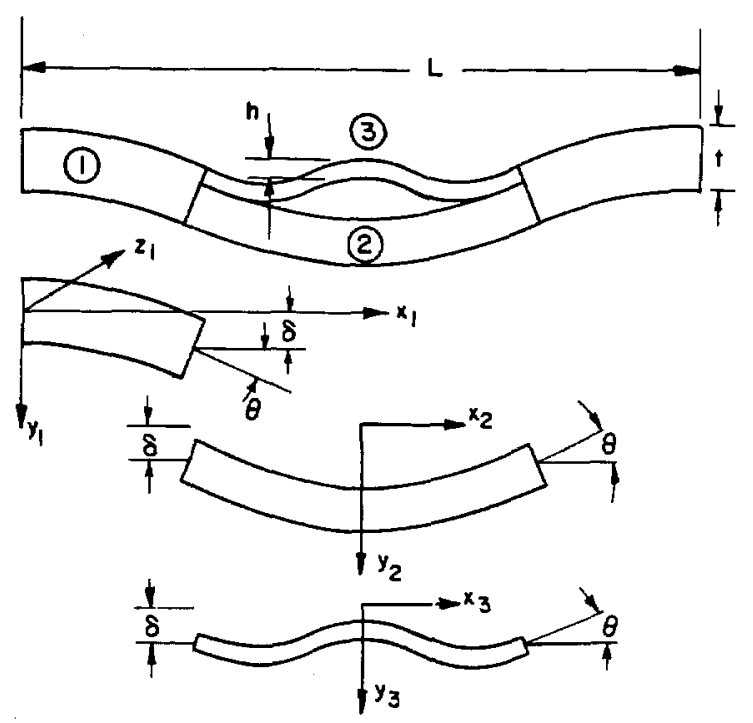

Fig. 7. Coordinate system for general panel problem. 
Equilibrium

$$
\text { At } x_{1}=l_{1}, x_{i}=-l_{i} / 2, i=2,3
$$

Shear

$$
S_{1}=S_{2}+S_{3}
$$

Axial Force

$$
P_{1}=P_{2}+P_{3}
$$

Moment

$$
M_{1}=M_{2}+M_{3}-P_{2} \frac{h}{2}+P_{3} \frac{t-h}{2}
$$

The shear condition (16) (with the aid of (17)) produces a relation between $\delta$ and $\theta$,

$$
\delta=\theta \frac{l_{1}}{2 u_{1}} \tan u_{1}
$$

Substituting this relation in (15) produces the following results for the deflections:

$$
\left.\begin{array}{l}
y_{1}=\frac{\theta l_{1}}{2 u_{1} \sin 2 u_{1}}\left(1-\cos 2 u_{1} x_{1} / l_{1}\right), \\
y_{i}=\frac{\theta l_{i}}{2 u_{i} \sin u_{i}}\left[\cos 2 u_{i} x_{i} / l_{i}-\cos 2 u_{i}\left\{\left\{\cos u_{i}\right\}\right], i=2,3\right.
\end{array}\right\}
$$

where $u_{i}=\left(l_{i} / 2\right) \vee\left(P_{i} \mid D_{i}\right), i=1,2,3$, are the normalized total loads and $D_{i}=\left(E t_{i}^{3} / 12\left(1-\nu^{2}\right)\right)$ are the stiffness rigidities of the separate sections.

The remaining conditions necessary for a solution involve the overall shortening of the plate. Assume that during the transition from state ii to state iii the ends remain fixed (i.e. overall shortening is $\epsilon_{0} L$ ). This produces the conditions essentially analogous to (3), namely

$$
\begin{gathered}
\epsilon_{0} L=2 \epsilon_{1} l_{1}+\int_{0}^{l_{1}}\left(\frac{\mathrm{d} y_{1}}{\mathrm{~d} x_{1}}\right)^{2} \mathrm{~d} x_{1}+\epsilon_{2} l_{2}+\frac{1}{2} \int_{-l_{2} / 2}^{l_{2} / 2}\left(\frac{\mathrm{d} y_{2}}{\mathrm{~d} x_{2}}\right)^{2} \mathrm{~d} x_{2}+h \theta, \\
\epsilon_{3} l_{3}+\frac{1}{2} \int_{-l_{3} / 2}^{l_{3} / 2}\left(\frac{\mathrm{d} y_{3}}{\mathrm{~d} x_{3}}\right)^{2} \mathrm{~d} x_{3}=\epsilon_{2} l_{2}+\frac{1}{2} \int_{-l_{2} / 2}^{l_{2} / 2}\left(\frac{\mathrm{d} y_{2}}{\mathrm{~d} x_{2}}\right)^{2} \mathrm{~d} x_{2}+t \theta,
\end{gathered}
$$

where $\epsilon_{i}$ is the midsurface strain in the " $i t h$ " segment and is related to the axial load $P_{i}$. The membrane stresses and strains are given by (plane strain assumption)

$$
\begin{aligned}
& \left(\sigma_{x}\right)_{i}=\frac{E}{\left(1-\nu^{2}\right)}\left(\nu^{2} \epsilon_{0}-\epsilon_{i}\right),\left(\sigma_{z}\right)_{i}=\frac{E \nu}{\left(1-\nu^{2}\right)}\left(\epsilon_{0}-\epsilon_{i}\right), \\
& \left(\epsilon_{z}\right)_{i}=\nu \epsilon_{0},\left(\epsilon_{x}\right)_{i} \equiv-\epsilon_{i}, i=1,2,3 .
\end{aligned}
$$

The strain energy in the system is then calculated as

$$
\begin{gathered}
U=\left[\left(\sigma_{x}\right)_{1}\left(\epsilon_{x}\right)_{1}+\left(\sigma_{z}\right)_{1}\left(\epsilon_{z}\right)_{1}\right] t_{1} l_{1}+D_{1} \int_{0}^{l_{1}}\left(\frac{\mathrm{d}^{2} y_{1}}{\mathrm{~d} x_{1}^{2}}\right)^{2} \mathrm{~d} x_{1} \\
+\frac{1}{2} \sum_{i=2}^{3}\left\{\left[\left(\sigma_{x}\right)_{i}\left(\epsilon_{x}\right)_{i}+\left(\sigma_{z}\right)_{i}\left(\epsilon_{z}\right)_{i}\right] t_{i} l_{i}+D_{i} \int_{-i, j}^{l, j 2}\left(\frac{\mathrm{d}^{2} y_{i}}{\mathrm{~d} x_{i}^{2}}\right)^{2} \mathrm{~d} x_{i}\right\} .
\end{gathered}
$$

Consider now $\epsilon_{1}, \epsilon_{2}, \epsilon_{3}$ and $\theta$ as the desired unknown quantities with load and section parameters specified. Combining eqn (19) with (17), (18), (20) and (21) produces four equations in the four unknowns. The nondimensional versions of these equations are given in the Appendix along with the energy.

The system of equations (A1)-(A4) in the Appendix cannot be solved in closed form and a numerical iterative scheme is employed. In order to start the iteration, an initial guess to the solution is required. The subject of finding such an initial guess is considered next. 
Numerical solution

Let $u_{3}$ represent the normalized load for Section 3 as defined in the nomenclature. Starting from the observation that for $\theta=0$ the post buckling normalized axial load $u_{3}$ is given by $u_{3}=\pi$, let us examine the equilibrium position for the more general case $\theta \neq 0$. Figure 8 shows the axial load defiection curve for specific values of $\theta$ as obtained from (19). Denote $y_{3}(0)-\delta$ by $A_{3}$ as in Fig. 8; then the third of $(19 ; \mathrm{i}=3)$ yields

$$
A_{3}=\frac{l_{3} \theta\left(1-\cos u_{3}\right)}{2 u_{3} \sin u_{3}}
$$

In the lower part of this figure, $u_{3}<\pi\left(A_{3}>0\right)$; this case corresponds to a closing of the delamination against Section 2 and is, therefore, of no current interest. Focusing attention on $u_{3}>\pi$, it is seen in Fig. 8 that for a given section geometry $u_{3}$ decreases with decreasing $\theta$, approaching the Euler buckling load $\left(u_{3}=\pi\right)$ as $\theta \rightarrow 0$. We shall make use of this observation later on. For the general case $\theta \neq 0$, let

$$
u_{3}=\pi+\eta, \quad 0<\eta<\pi .
$$

Substitution of (24) in the equations determining $\epsilon_{1}, \epsilon_{2}, \epsilon_{3}$ and $\theta$ (see eqns (Ai) to (A4) in the appendix) and the third of (A5) in the appendix, results in eight equations with eight unknowns

$$
\left.\begin{array}{l}
\bar{\epsilon}_{1}=\left[(1-\bar{h}) \bar{\epsilon}_{0}+\bar{h} \bar{\epsilon} \bar{\epsilon}_{3}^{0}\right] / \kappa+e_{1}, \quad u_{1}=\pi(1-\bar{l})\left[\left(\bar{\epsilon}_{1}-\nu^{2} \bar{\epsilon}_{0}\right)\left(1-\nu^{2}\right)\right]^{1 / 2} / 2 \\
\bar{\epsilon}_{2}=\left[\bar{\epsilon}_{0}-\bar{h}(1-\bar{l}) \bar{\epsilon}_{3}^{0}\right] / \kappa+e_{2}, \quad u_{2}=\pi \bar{l}\left[\left(\bar{\epsilon}_{2}-\nu^{2} \bar{\epsilon}_{0}\right) /\left(1-\nu^{2}\right)\right]^{1 / 2} /(1-\bar{h}) \\
\bar{\epsilon}_{3}=\bar{\epsilon}_{3}^{0}+e_{3}, \quad \bar{\epsilon}_{3}^{0}=\left(1-\nu^{2}\right) \bar{\epsilon}_{\mathrm{cr}}+\nu^{2} \bar{\epsilon}_{0} \\
\bar{\theta}=\frac{-\frac{(1-\bar{h})}{\bar{l}}\left[\left(\frac{2 u_{1} \bar{l}}{1-\bar{l}}\right)^{2}-\left\{u_{2}(1-\bar{h})\right\}^{2}\right]-2 \pi^{2} \bar{h}^{3}\left[\left(\bar{\epsilon}_{0}-\bar{\epsilon}_{\mathrm{cr}}+a_{1}\right) / 3 \kappa\right]^{1 / 2}\left(1+a_{2}\right)}{[\bar{l} /(1-\bar{l})] 2 u_{1} / \tan 2 u_{1}+\left(1-\bar{h}^{3} u_{2} / \tan u_{s}\right.} \\
\eta=\bar{\theta}\left[\left(\bar{\epsilon}_{0}-\bar{\epsilon}_{\mathrm{cr}}+a_{1}\right) 4 \pi^{2} / 3 \kappa\right]^{-1 / 2}
\end{array}\right\}
$$

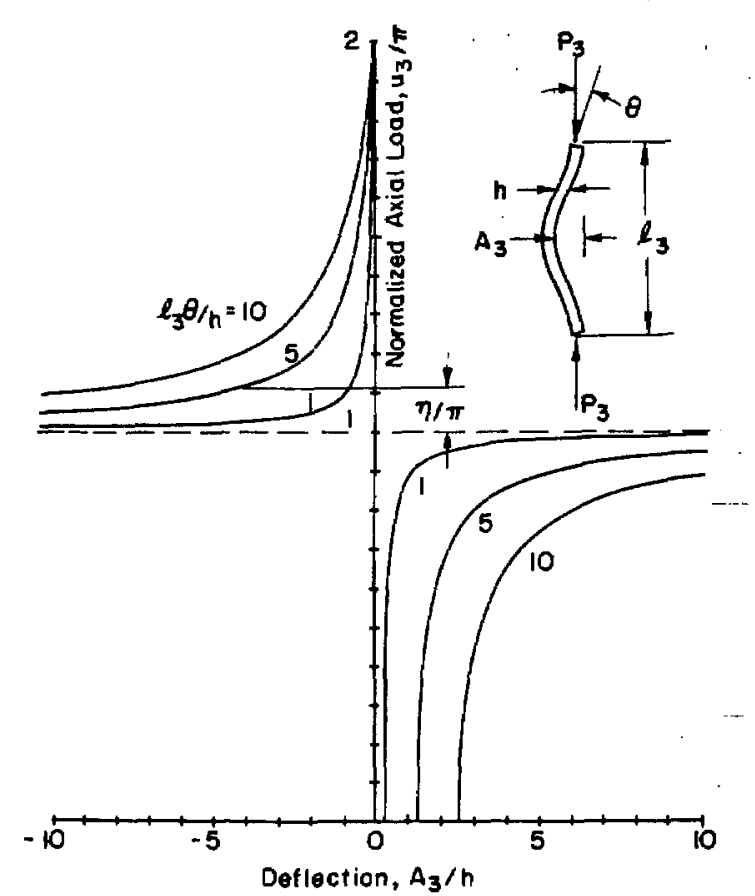

Fig. 8. Equilibrium position of Section 3. 
where terms defined in the listing of the nomenclature are used. Also $\kappa=1-\bar{h}+\bar{h} \bar{l}$ and the quantities $e_{1}, e_{2}, e_{3}, a_{1}$ and $a_{2}$ are given in the appendix. Let us seek a first approximation to the solution by assuming that $\theta$ is small $(\theta \ll 1)$. This implies (from the discussion of Fig. 8) that $\eta$ is also small, from which it follows that the quantities, $e_{1}, e_{2}, e_{3}, a_{1}$ and $a_{2}$ are small.

By temporarily assuming zero values for these quantities an initial solution to the set (25) can now be calculated, which, in turn, allows the calculation of the quantities $e_{1}, e_{2}, e_{3}, a_{1}$ and $a_{2}$. Successive iterations can now be carried out (about five) until sufficient convergence is achieved.

Next, the strain energy is calculated from (A6) from which, by means of simple numerical differentiation, the strain energy release rate can be found.

The results obtained in this manner are shown in Fig. 9, where the non-dimensionalized strain energy release rate $\bar{G}_{e}$ for "model e" of Fig. 2 is plotted as a function of the crack length for several loadings and section dimension. Consider a typical curve in Fig. 9 which corresponds to a fixed load ratio, $\bar{\epsilon}_{0}$, and examine the characteristic behavior of $\bar{G}_{e}$ with crack length ratio $\bar{l}_{\text {. }} \bar{G}_{e}$ is nonzero (positive) only when $\bar{l}>\bar{l}_{\mathrm{cr}}$ where $\bar{l}_{\mathrm{cr}}$ is the critical buckling length given by $\bar{l}_{\mathrm{cr}}=\bar{h} / \sqrt{ }\left(\bar{\epsilon}_{0}\right) . \bar{G}_{e}$ increases rapidly with delamination length reaching a maximum at $\| L=\bar{l}_{e^{*}}$. Its minimum occurs at $\| L=\bar{l}_{e^{* * *}}$. The difference between the minimum $\bar{G}_{e}\left(\bar{l}_{e^{* *}}\right)$ and maximum $\bar{G}_{e}\left(\bar{l}_{e^{*}}\right)$ is more striking for this case than that for the thin film case. This is significant in that these values dictate the region of stable delamination growth as shown in the discussion of Figs. 5 and 6.

A great simplification in the general problem just treated can be achieved by neglecting bending contributions of the sections' structure other than section 3 , i.e. by assuming $\theta \equiv 0$. This leads to model $\mathrm{b}$ (Fig. 2b). The condition $\theta=0$ implies $e_{1}=e_{2}=e_{3}=a_{1}=a_{2}=\eta=0$ in (25), (while $\eta / \theta$ is finite). By substituting the reduced results of (25) in (A6) while taking the limit as $u_{3} \rightarrow \pi$ we find:

$$
\bar{U}=-\pi^{4} \bar{h} \bar{l}\left(\bar{\epsilon}_{0}-\bar{\epsilon}_{\mathrm{cr}}\right)^{2} /(18 \kappa)+\pi^{4} \bar{\epsilon}_{0}^{2} /\left\{18\left(1-\nu^{2}\right)\right\}
$$

The strain energy release rate, $G_{b}$, is given by $\left(G_{b}=-\partial U / \partial l\right)$. Thus

$$
\bar{G}_{b}=\pi^{4} \bar{h}(1-\bar{h})\left(\bar{\epsilon}_{0}-\bar{\epsilon}_{\mathrm{cr}}\right)\left[\bar{\epsilon}_{0}+\bar{\epsilon}_{\mathrm{cr}}\{3+4 \bar{h} \bar{l} /(1-\bar{h})\}\right] /\left(18 \kappa^{2}\right) .
$$

Generalization of this model with $n$ (integer) delaminations results in model $2 \mathrm{c}$, the analysis of which is identical to that of model $2 \mathrm{~b}$ if $h$ is replaced by $n h$ (while leaving $\epsilon_{\mathrm{cr}}$ unchanged). Thus, from (27)

$$
\bar{G}_{c}=\pi^{4} n \bar{h}(1-n \bar{h})\left(\bar{\epsilon}_{0}-\bar{\epsilon}_{\mathrm{cr}}\right)\left[\bar{\epsilon}_{0}+\bar{\epsilon}_{\mathrm{cr}}[3+4 n \bar{h} \bar{l}(1-n \bar{h})\}\right] /\left\{18(1-n \bar{h}+n \bar{h} \bar{l})^{2}\right\}
$$

The symmetric split (model d) is a particular case of model $2 \mathrm{c}$ with $n=2, \bar{h}=0.5(n \bar{h} \rightarrow 1)$. This gives

$$
\bar{G}_{d}=\pi^{4}\left[\bar{\epsilon}_{0}-1 /\left(4 \bar{l}^{2}\right)\right] /\left(18 \bar{l}^{3}\right) .
$$

Finally, it is pointed out that the "thin film" model (model a) treated earlier is a further simplification of the general case over case b with $\bar{h}=h / t \rightarrow 0$

Thus

$$
\bar{G}_{a}=h \lim _{\bar{h} \rightarrow 0}\left(\bar{G}_{b} / h\right)
$$

where $G_{a}$ and $G_{b}$ are given in (8) and (27) respectively.

Three of the models are compared in Fig. 9. It can be seen there that the "thick beam" model is not a great improvement over the "thin film" model. The range of applicability of the "thin film" model can be established by comparing measures such as the maximum value of energy release rate. This is done in Fig. 10 up to a delamination thickness ratio of 0.10 . Over this range the error between the two models is montonic with $h / t$ and load $\left(\epsilon_{0} / \epsilon_{L}\right)$. For larger values of $h / t$ the comparison becomes more complicated due to the large shifts in the position of the maximum energy release rate. 


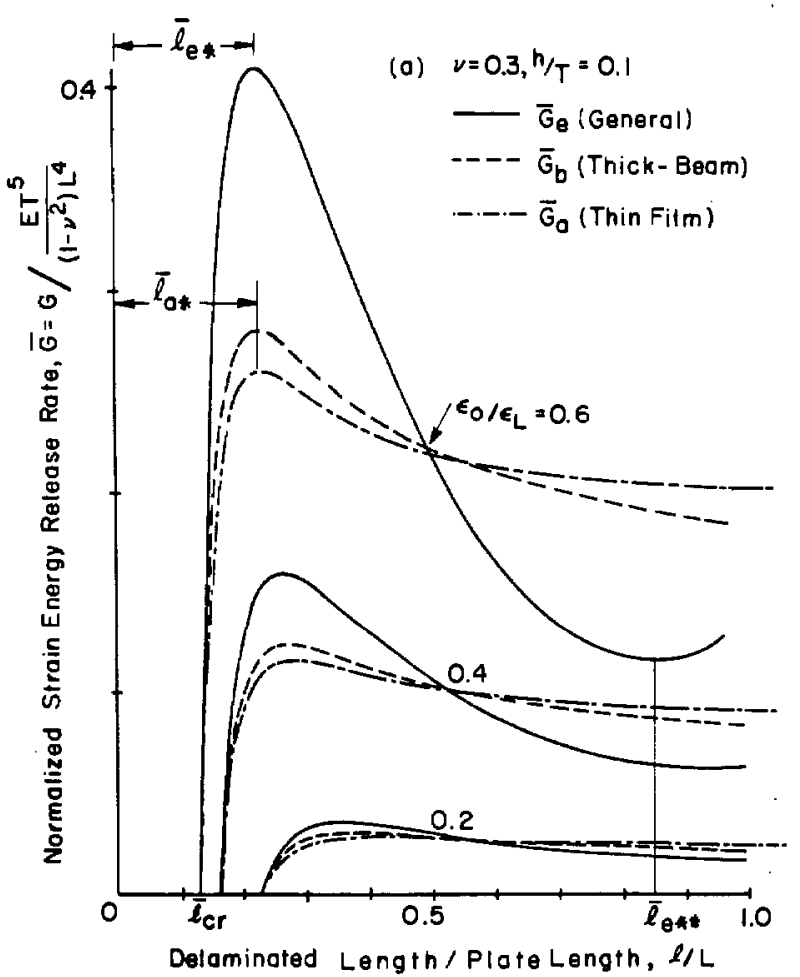

(a)

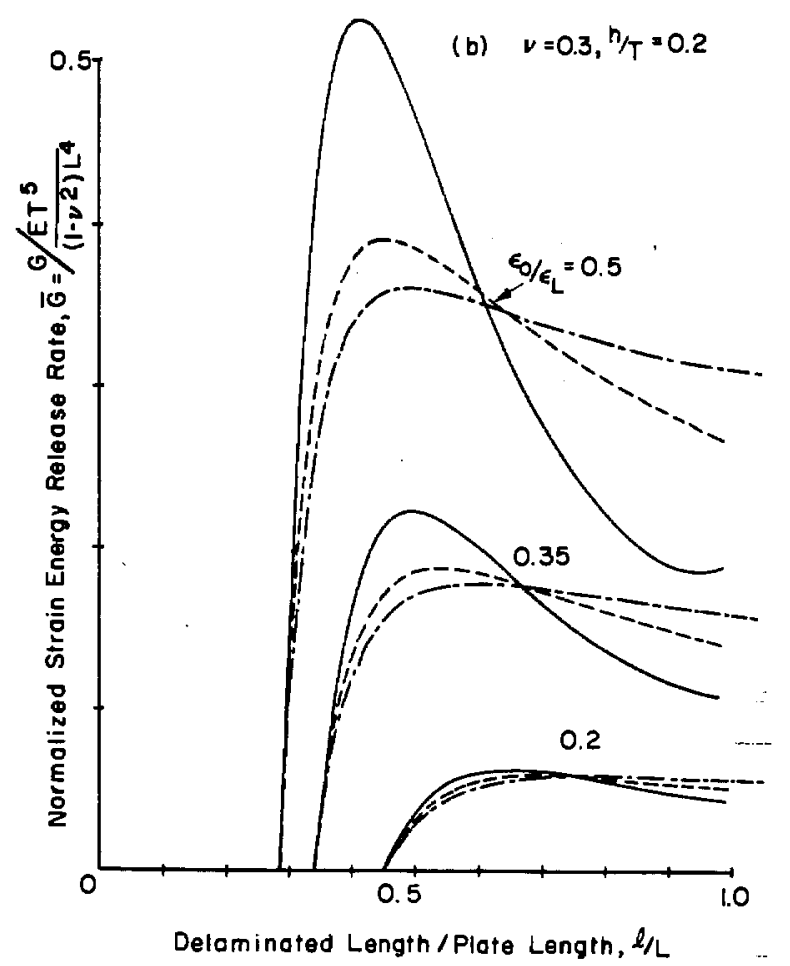

(b)

Fig. 9. Strain energy release rate-various models. 


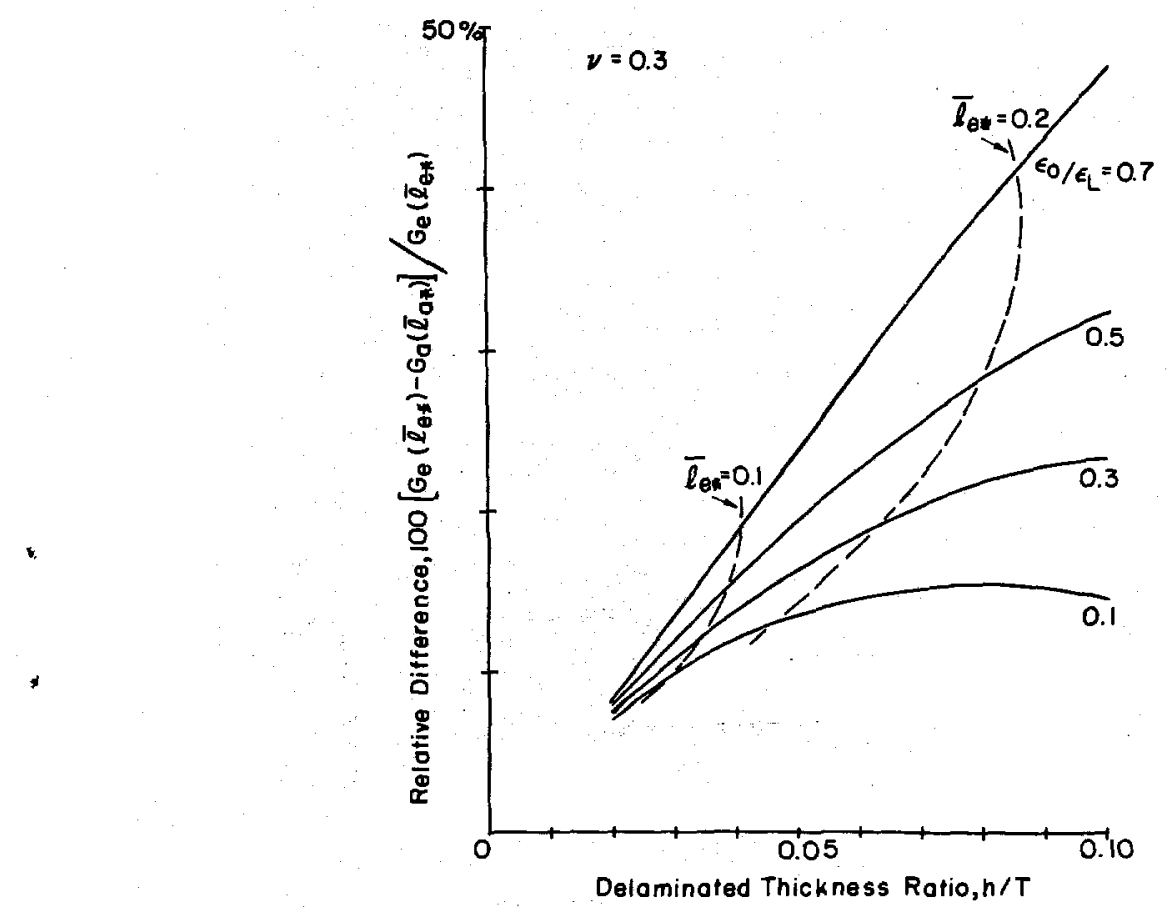

Fig. 10. Relative difference between thin film and general model as a function of the controlling parameters.

\section{SUMMARY AND CONCLUSIONS}

The models of delamination buckling and growth introduced in this paper display an interesting variety of behavior depending upon the dimensions of the delamination, the load at which it is introduced and the fracture energy. When loading an initially delaminated structure, the growth of the delamination may be stable, unstable or an unstable growth followed by a stable growth. The range of this behavior can be found from the results presented and could form the base for an experimental study of the applicability of the proposed model.

The solution of the case when the delamination is introduced in a preloaded structure awaits the resolution of the problem of the excess energy released in going from the unbuckled to the buckled state. However, the model does show the magnitude of this excess energy which can participate in the fracture process. This energy excess would lower the "load" necessary to initiate growth. This finding is consistent with experimental evidence [6] but quantitative comparison of the present analysis with impact experiments is not very meaningful because the present analog study is hardly capable of dealing with the details of the complex dynamic process of delamination growth in an impacted plate.

The "general" model considered here for the delamination buckling and growth is useful in establishing the range of validity of the simplified models (e.g. "thin film" case). This is important in that an extension of the model to two dimensions (growth in both the longitudinal and transverse directions) is very difficult for the analog of the present "general" model but tractable for the "thin film" case.

Acknowledgements-This work was supported by the National Aeronautics and Space Administration under Grant NSG-1483. The authors are appreciative of this support and wish to acknowledge the encouragement and interest of $\mathrm{Dr}$. James H. Starnes, Jr., NASA Langley, in pursuing this work.

\section{REFERENCES}

1. M. D. Rhodes, J. G. Williams and J. H. Starnes, Jr., Effect of impact damage on the compression strength of filamentary-composite hat-stiffened panels: presented at the 23 rd SAMPE National Symp. and Exhibition, Anaheim, California (2-4 May 1978), SAMPE Vol. 23, Selective application of materials for products and energy, pp. 300-319 (May 1978).

2. M. D. Rhodes, J. G. Williams and J. H. Starnes, Jr,, Effect of low-velocity impact damage on the compressive strength of graphite-epoxy hat-stiffened panels. NASA Tech. Note D-8411 (April 1977). 
3. J. H. Starnes, Jr., M.D. Rhodes and J. G. Williams, The effect of impact damage and circular.holes on the compressive strength of a graphite-epoxy laminate. Nondestructive Evaluation and Flaws Criticality for Composite Materials ASTM STP 696 (Edited by R. B. Pipes), pp. 145-171. ASTM (1979).

4. J. G. Williams, M. S. Anderson, M.D. Rhodes, J. H. Starncs, Jr. and W. J. Stroud, Recent developments in the design, testing and impact-damage tolerance of stiffened composite panels. NASA Tech. Memo. 80077 (April 1979).

5. M. M. Mikulas, Jr., H. G. Bush, M.D. Rhodes, Current Langley Research Center studies on buckling and low velocity impact of composite panels. 3rd Conf. on Fibrous Composites in Flight Vehicle Design, Part II, NASA TM X-3377, pp. $633-663(1976)$.

6. M. D. Rhodes, J. G. Williams and J. H. Starnes, Jr., Low-velocity impact damage in graphite-fiber reinforced epoxy laminates, presented at the 34th Ann. Conf. on Reinforced Plastics/Composite Institute, New Orleans, Louisiana (29 Jan. 2 Feb 1979).

7. W. G. Knauss, C. D. Babcock and H. Chai, Visualization of impact damage of composite plates by means of the Moire technique. NASA CR-159261 (April 1980).

8. H. Chai, C. D. Babcock and W. G. Knauss, Fracture encrgy of graphite-epoxy composite laminates. Galcit SM Rep. 79-13, July 1979, (Solid Mechanics publication, California Institute of Technology).

9. H. Chai, C. D. Babcock and W. G. Knauss, On the interaction of buckling and delamination. Galcit SM Rep. 79-14, Sept. 1979, (Solid Mechanics publication, California Institute of Technology).

10. H. Chai, C. D. Babcock and W. G. Knauss, The incorporation of bending into the buckling delamination analysis. Galcit SM Rep. 79-15, Nov. 1979, (Solid Mechanics publication, California Institute of Technology).

11. H. Chai, C. D. Babcock, W. G. Knauss, Interaction of buckling and delamination in a delaminated beam under pure bending. Galcit SM Rep. 79-16, Dec. 1979, (Solid Mechanics publication, California Institute of Technology)

12. L. M. Kachanov, Separation of composite materials, Mekh. Polim. No, 5, 918-922 (1976).

13. L. M. Kachanov, The separation of fiberglass tubes subjected to an external pressure. Mekh. Polim. No. 6, 1106-1108 (1975).

\section{APPENDIX}

The $f_{i}$ and $g_{i}$ in eqn (15) are as follows:

$$
\begin{aligned}
f_{1}(x) & =\frac{l_{1}}{u u 1\left(1-u_{1} / \tan u_{1}\right)}\left[\frac{\left(\sin 2 u_{1}-2 u_{\mathrm{k}}\right)}{\sin ^{2} u_{1}} \sin ^{2} u_{1} x_{1} / l_{1}-\sin 2 u_{1} x_{1} / l_{1}+2 u_{1} x_{1} / l_{1}\right] \\
g_{1}(x) & =\frac{1}{2\left(1-u_{1} / \tan u_{1}\right)}\left[\left(\sin 2 u_{1} x_{1} / l_{1}-2 u_{i} x_{1} / l_{1}\right) / \tan u_{1}+2 \sin ^{2} u_{1} x_{1} / l_{1}\right] \\
f_{i}(x) & =\frac{l_{i}}{2 u_{i} \sin u_{i}}\left(\cos 2 u_{i} x_{i} / l_{i}-\cos u_{i}\right) \\
g_{i}(x) & =1, \quad i=2,3 \\
u_{i} & =\frac{l_{i}}{2} V\left(P_{i} / D_{i}\right), \quad i=1,2,3 .
\end{aligned}
$$

The nondimensional equations for $\xi_{i}$ and $\theta$ are

$$
\begin{aligned}
& \bar{\epsilon}_{1}-(1-\bar{h}) \bar{\epsilon}_{2}-\bar{h} \bar{\epsilon}_{3}=0 \\
& \left\{\frac{3 \bar{h} \bar{\theta}}{\pi^{2}}+\frac{3 \bar{\theta}^{2}}{8 \pi^{2}}\left[\frac{(1-\bar{l})\left(4 u_{1}-\sin 4 u_{1}\right)}{2 u_{1} \sin ^{2} 2 u_{1}}+\frac{\bar{l}\left(2 u_{2}-\sin 2 u_{2}\right)}{u_{2} \sin ^{2} u_{2}}\right]\right\} \\
& +\frac{1}{\left(1-v^{2}\right)}\left[\bar{\epsilon}_{1}-\bar{\epsilon}_{0}+\left[\left(\bar{\epsilon}_{2}-\bar{\epsilon}_{1}\right)\right]=0\right. \\
& \left\{\frac{3 \bar{\theta}}{\pi^{2} \bar{l}}+\frac{3 \bar{\theta}^{2}}{8 \pi^{2}} \frac{\left(2 u_{2}-\sin 2 u_{2}\right)}{u_{2} \sin ^{2} u_{2}}\right\}-\frac{3 \bar{\theta}^{2}}{8 \pi^{2}} \frac{\left(2 u_{3}-\sin 2 u_{3}\right)}{u_{3} \sin ^{2} u_{3}}+\frac{\left(\bar{\epsilon}_{2}-\bar{\epsilon}_{3}\right)}{\left(1-\nu^{2}\right)}=0 \\
& \bar{\theta} \bar{l}\left[\frac{\bar{l}}{(1-\bar{l})} 2 u_{1} / \tan 2 u_{1}+(1-\bar{h})^{3} u_{2} / \tan u_{2}+\bar{h}^{3} u_{3} / \tan u_{3}\right] \\
& +(1-\bar{h})\left\{\left[\frac{2 u_{1} \bar{l}}{(1-\bar{l})}\right]^{2}-\left[\left.(1-\bar{h}) u_{2}\right|^{2}\right\}=0\right. \\
& u_{1}=\pi(1-\bar{T}) \vee\left(\left(\bar{\epsilon}_{1}-\nu^{2} \bar{\epsilon}_{0}\right) /\left(1-\nu^{2}\right)\right) / 2 \\
& u_{2}=\pi \bar{l} V\left(\left(\bar{\epsilon}_{2}-\nu^{2} \bar{\epsilon}_{0}\right) /\left(1-\nu^{2}\right)\right) /(1-\bar{h}) \\
& u_{3}=\pi \bar{l} V\left(\left(\bar{\epsilon}_{3}-\nu^{2} \bar{\epsilon}_{0}\right) /\left(1-\nu^{2}\right)\right) / \bar{h} \text {. }
\end{aligned}
$$


The nondimensional strain energy is as follows;

$$
\begin{gathered}
U /\left\{E T^{3} L^{-3}\left(1-\nu^{2}\right)^{-1}\right\}=\bar{U} \\
\bar{U}=\frac{\pi^{4}}{\left[8\left(1-v^{2}\right)^{2}\right.}\left[(1-\bar{l}) \bar{\epsilon}_{1}^{2}+(1-\bar{h}) \overline{\epsilon_{2}}{ }^{2}+\bar{h}_{\bar{\epsilon}} \bar{\epsilon}_{3}^{2}+\nu^{2} \bar{\epsilon}_{0}\left(\bar{\epsilon}_{0}-2 \bar{\epsilon}_{1}\right)\right] \\
+\frac{\bar{\theta}^{2}}{24}\left[\frac{2}{(1-\bar{l})} \frac{u_{1}\left(4 u_{1}+\sin 4 u_{1}\right)}{\sin ^{2} 2 u_{1}}+\frac{(1-\bar{h})^{3}}{\bar{l}} \frac{u_{2}\left(2 u_{2}+\sin 2 u_{2}\right)}{\sin ^{2} u_{2}}\right. \\
\left.+\frac{\bar{h}^{3}}{\frac{u_{3}\left(2 u_{3}+\sin 2 u_{3}\right)}{\sin ^{2} u_{3}}}\right]
\end{gathered}
$$

The $e_{j}, i=1,2,3 ; a_{1}$ and $a_{2}$ in eqn (25) are as follows;

$$
\begin{gathered}
e_{1}=-\left[\left(1-\nu^{2}\right)(1-\bar{h}) \alpha_{1}-\bar{h} \bar{l}_{3}\right] / \kappa \\
e_{2}=-\left[\left(1-\nu^{2}\right) \alpha_{1}+\bar{h}(1-\bar{l}) e_{3}\right] / \kappa \\
e_{3}=2\left(1-\nu^{2}\right) \bar{\epsilon}_{\mathrm{cr}}\left(\eta / \pi+\eta^{2} / 2 \pi^{2}\right) \\
a_{1}=\alpha_{2} \kappa-\alpha_{1}-e_{3} /\left(1-\nu^{2}\right)-\frac{3 \kappa}{4 \pi^{2}}\left(\frac{\bar{\theta}}{\sin \eta}\right)^{2}\left[1-\left(\frac{\sin \eta}{\eta}\right)^{2}-\frac{\sin 2 \eta}{2(\pi+\eta)}\right] \\
a_{2}=(\eta / \tan \eta) \cdot(1+\eta / \pi)-1
\end{gathered}
$$

Where $\alpha_{1}$ and $\alpha_{2}$ are given by the curley bracket of eqns (A2) and (A3) respectively and $x=1-\bar{h}+\bar{h} \bar{l}$. 